\title{
Modelos de Ecuaciones Estructurales: Características, Fases, Construcción, Aplicación y Resultados
}

\section{STRUCTURAL EQUATION MODELING: FEATURES, PHASES, CONSTRUCTION, IMPLEMENTATION AND RESULTS}

\author{
María Teresa Escobedo Portillo', Jesús Andrés Hernández Gómez'1, Virginia Estebané Ortega', Guillermina Martínez Moreno
}

1. Universidad Autónoma de Ciudad Juárez, Chihuahua, México.

\begin{abstract}
RESUMEN
Se exponen las características y fases de los modelos de ecuaciones estructurales, así como las etapas de construcción, siendo estas la especificación, identificación, estimación de parámetros, evaluación del ajuste, reespecificación del modelo y la interpretación de resultados. Se presenta el análisis factorial exploratorio y confirmatorio como parte para la construcción de un modelo. Se detallan algunos paquetes computacionales como el LISREL, AMOS y SPSS. A modo de ejemplificar los modelos de ecuaciones estructurales, se realiza un estudio en la población docente de tiempo completo del departamento de ingeniería Industrial y Manufactura de una universidad pública de Ciudad Juárez, Chihuahua, México. Se estudia el impacto de los factores socioculturales y ergoambientales en la satisfacción laboral, obteniendo un modelo resultante en el que se detallan las variables más y menos significativas. No se rechazan las cuatro hipótesis cuyos valores son $0,06,0,06,0,071,0,074$, respectivamente.
\end{abstract}

(Escobedo M, Hernández J, Estebané V, Martínez G, 2016. Modelos de Ecuaciones Estructurales: Características, Fases, Construcción, Aplicación y Resultados. Cienc Trab. Ene-Abr; 18 [55]: 16-22).

Palabras clave: MODELOS ESTRUCTURALES, FACTORES ERGOAMBIENTALES, FACTORES SOCIOCULTURALES, SATISFACCIÓN LABORAL.

\section{ABSTRACT}

The characteristics and phases of structural equation modeling and construction phases, and these are the specification, identification, parameter estimation, assessment of adjustment, re-specification of the model and the interpretation of results are shown. The exploratory and confirmatory factor analysis as part for the construction of a model is presented. Some software packages like LISREL, AMOS and SPSS are detailed. By way of illustrating the structural equation modeling, a study was performed on the population of full-time faculty of the Department of Industrial Engineering and Manufacturing of a public university in Ciudad Juarez, Chihuahua, Mexico. The impact of sociocultural and ergoambientales factors in getting a job satisfaction resulting model in which more and less significant variables detailed studied. The four scenarios whose values are 0,06 , $0,06,0,071,0,074$ respectively can not refuse.

Key words: STRUCTURAL MODELS, ERGOENVIRONMENTAL FACTORS, SOCIOCULTURAL FACTORS, JOB SATISFACTION.

\section{INTRODUCCIÓN}

Para efectos de ejemplificar la teoria respectiva al tema en desarrollo, se presentan en las secciones correspondientes una investigación en la que se evalúa el impacto de los Factores Socioculturales (FSC) y los Factores Ergoambientales (FEA) en la Satisfacción Laboral (SL) del personal académico del Departamento de Ingeniería Industrial y Manufactura de una Institución de Educación Superior en Ciudad Juárez, Chihuahua.

Los modelos de ecuaciones estructurales establecen la relación de dependencia entre las variables. Trata de integrar una serie de

Correspondencia / Correspondence:

María Teresa Escobedo Portillo

Departamento de Ingenieria Industrial

IIT/UACJ

Tel: 6884800 ext. 5458

e-mail: mayteesc@gmail.com

Recibido: 01 de Septiembre de 2015 / Aceptado: 28 de 0ctubre de 2015

ecuaciones lineales y establecer cuáles de ellas son dependientes o independientes de otras, ya que dentro del mismo modelo las variables que pueden ser independientes en una relación pueden ser dependientes en otras ${ }^{1}$, por lo que se vuelve una herramienta útil. $^{2}$

Estos modelos surgen de la necesidad de encontrar la flexibilidad en los modelos de regresión. ${ }^{3}$ Desde inicios del siglo XX hasta 1970, los investigadores fueron aportando conocimiento para la creación de dichos modelos, hasta que Jöreskog, ${ }^{4}$ presenta la primera formulación del Análisis de la Estructura de Covarianzas (CSA) -por sus siglas en inglés-, el mismo que más tarde se conoció como LISREL. ${ }^{5}$ De igual manera, estos modelos nacen de unir dos tradiciones ${ }^{6}$, la predicción y el enfoque psicométrico. Estos modelan conceptos en variables latentes (no observadas) e infieren en múltiples medidas observadas (variables manifestadas). La técnica de SEM se considera una extensión de varias técnicas multivariantes de regresión múltiple, el análisis factorial principalmente y el análisis de senderos. ${ }^{7}$ Abarcan varios modelos conocidos por muchos nombres, entre ellos el análisis de la estructura de covarianza, análisis de la variable latente, análisis factorial confirmatorio ${ }^{8}$, e incluso el análisis LISREL. ${ }^{9}$

En general los SEM permiten, primeramente, crear modelos del error de medida. En otras palabras, el grado en que las variables se 
pueden medir. ${ }^{10}$ Después, incorpora constructos abstractos e inobservables (variables latentes y variables teóricas no observadas). También modela las relaciones entre múltiples variables predictoras (independientes o exógenas) y variables criterio (dependientes o endógenas).

Combina y confronta el conocimiento a priori e hipótesis con datos empíricos, por lo que los modelos de ecuaciones estructurales son más confirmatorios que exploratorios. ${ }^{11}$

Figura 1.

Modelo causal propuesto.

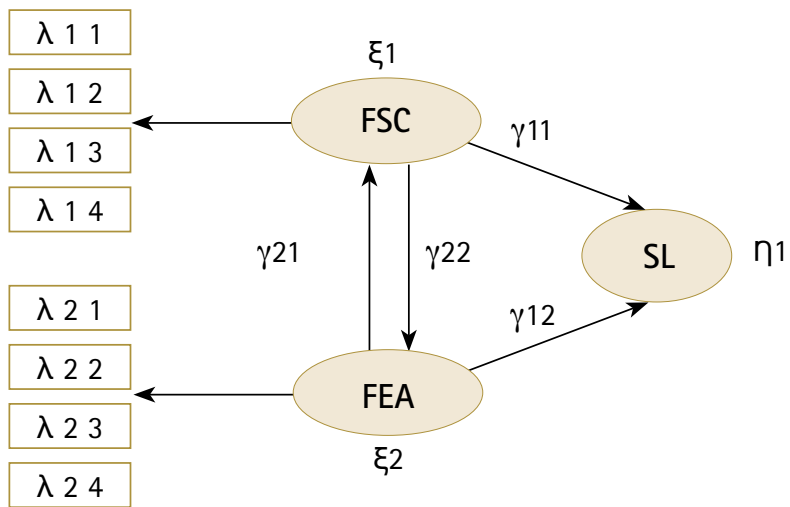

Fuente: elaboración propia.

En la Figura 1 se observa el modelo causal propuesto para el ejemplo presentado, el mismo que indica las variables latentes tanto endógenas ( $\mathrm{n} 1)$ como la SL, y las exógenas como los FSC (६1) y los FEA (६2). Dentro de las variables observadas endógenas se encuentran $\lambda 11$ (Edad), $\lambda 12$ (Género), $\lambda 13$ (Último grado de estudios y $\lambda 14$ (Estado civil), $\lambda 21$ (Iluminación natural), $\lambda 22$ (Iluminación artificial), $\lambda 2$ 3(Ruido) y $\lambda 24$ (Temperatura).

Las relaciones entre constructos están determinadas por $\gamma 11$ (FSC - SL), $\gamma 12$ (FEA-SL), $\gamma 21$ (FSC-FEA) y $\gamma 22$ (FEA-FSC). Las anteriores pueden señalarse como hipótesis dependiendo de las necesidades tanto de la investigación como de los profesionales que lo llevan a cabo.

\section{CARACTERISTICAS DE LOS MODELOS DE ECUACIONES ESTRUCTURALES}

Los SEM se caracterizan por dos elementos principales. El primero, evaluar las relaciones de dependencia tanto múltiple como cruzadas. ${ }^{12}$ El segundo, el grado para representar conceptos no observados en estas relaciones y tener en cuenta el error de medida en el proceso de estimación. ${ }^{8}$

El sistema de ecuaciones estructurales tiene la ventaja, sobre otros sistemas y técnicas multivariantes, el analizar las relaciones por cada subconjunto de variables, permitiendo también una interrelación entre variables de diferentes grupos ${ }^{6}$, dependiendo del propósito de la investigación.

Los SEM trabajan con variables observables o medibles (aquellas que tienen un valor de entrada) y una o varias variables latentes o no observadas (que no tiene valor como tal y que puede utilizarse como un concepto), fortaleciendo las correlaciones utilizadas y realizando estimaciones más precisas de los coeficientes estructurales. ${ }^{2}$

Para el ejemplo que se presenta, se tienen las variables pertenecientes a la SL como son: percepción, beneficios, puesto, recono- cimiento, relación con compañeros, supervisión del jefe, condiciones de trabajo y libertad de acción. Referentes a los FEA se tienen las variables de ruido, iluminación natural, iluminación artificial y temperatura. Respecto a los FSC se cuenta con la edad, género, estado civil y último grado de estudios.

\section{FASES DE UN MODELO SEM}

Los especialistas en SEM coinciden en que son seis las fases para aplicar esta técnica. ${ }^{13,14}$ a) La especificación, b) identificación, c) estimación de parámetros, d) evaluación del ajuste, e) reespecificación del modelo y f) la interpretación de resultados lo conforman. Se expone una breve descripción de cada fase de acuerdo a Cupani. ${ }^{15}$

a) La especificación es la fase en donde el investigador establece la relación hipotética entre las variables latentes y las observadas, la misma que con el análisis se obtendrán las relaciones correctas.

b) En la fase de identificación se estiman los parámetros del modelo. Se determina si un modelo está identificado mediante una expresión algebraica que lo demuestre, en función de las varianzas y covarianzas muestrales.

c) En esta fase se determinan los valores de los parámetros desconocidos así como su respectivo error de medición para lo que se utilizan diversos programas computacionales como el LISREL, el AMOS y el EQS. ${ }^{9,16,17}$

d) La evaluación o bondad de ajuste se refiere a la exactitud en los datos del modelo para determinar si es correcto y sirve para los propósitos del investigador. Las medidas de calidad del ajuste pueden ser de tres tipos: (1) medidas absolutas del ajuste que evalúan el ajuste global del modelo, (2) medidas del ajuste incremental que comparan el modelo propuesto con otros modelos especificados por el investigador, o (3) medidas del ajuste de parsimonia, que ajustan las medidas de ajuste para ofrecer una comparación entre modelos con diferentes números de coeficientes estimados, siendo su propósito determinar la cantidad del ajuste conseguido por cada coeficiente estimado. ${ }^{18}$

En este punto se emplean indicadores para evaluar el ajuste del modelo. ${ }^{19}$ El más utilizado es cuando pvalue $>0,05$ y el RMSEA (error cuadrático medio de aproximación) $<0,05.20$ Siempre y cuando se asocien a una hipótesis.

e) La reespecificación del modelo ayuda al investigador a saber si el primer modelo obtenido es el mejor, para lo que es necesario buscar métodos para mejorar el ajuste del mismo añadiendo o eliminando los parámetros estimados del modelo original, con sus justificaciones correspondientes. Para tal caso, el valor del índice de modificación corresponde a la reducción del valor de chi-cuadrado, el cual se sugiere en un mínimo de 3,84 para ser significativa. ${ }^{18}$

f) La interpretación de los datos ayuda al investigador a establecer el modelo correcto y la aceptación o rechazo de las hipótesis, concluyendo con su investigación.

\section{Paquetes estadísticos para SEM}

Dado que los modelos de ecuaciones estructurales se crean a partir de parámetros estadísticos, se han diseñado varios paquetes computacionales que permiten calcular y analizar las relaciones entre variables. Entre estos se encuentra el AMOS (Analysis of Moment Structures, Análisis de estructuras de momento), ${ }^{16}$ el cual utiliza el 
modelado de ecuaciones estructurales para confirmar y explicar los modelos conceptuales que tratan las actitudes, percepciones y otros factores que determinan el comportamiento de las variables.

Este programa trabaja bajo la plataforma del SPSS (Statistical Package for the Social Sciences), el cual es un sistema global para el análisis estadístico de datos y con el que se pueden adquirir datos de casi cualquier tipo de archivo y utilizarlos para generar informes tabulares, gráficos y diagramas de distribuciones $\mathrm{y}$ tendencias, estadísticos descriptivos y análisis estadísticos complejos, permitiendo que el análisis estadístico sea accesible a cualquiera que lo necesite. ${ }^{21}$ Se puede emplear de manera interactiva o procesando muchas tareas a la vez. El trabajo interactivo se realiza a través del cuadro de dialogo que contiene los aspectos más importantes de la tarea que se vaya a realizar. Está organizado a base de comandos, como elementos de un lenguaje. Dichos comandos pueden ser generales o de aplicaciones estadísticas. ${ }^{22}$

Otro paquete es el LISREL (Lineal Structural Relations) creado por Jöreskog y Sörborn' ${ }^{9}$, el cual ofrece una mayor variedad de métodos de estimación y cuenta con una interfaz gráfica que permite crear el modelo (path diagram) automáticamente, una vez que se "corre" el análisis estadístico de los datos.

El EQS (Abreviatura de Equations), creado por Bentler (1985) ${ }^{17}$, tiene gran aceptación entre los investigadores que trabajan con modelos de ecuaciones estructurales.

\section{APLICACIÓN DE LOS SEM}

Son muchos los investigadores que han adoptado los modelos de ecuaciones estructurales para obtener resultados. Algunos de ellos comienzan por indagar y publicar desde la teoría, el origen y la estructura de estos: Mateos y Morales, Silva y Schiattino, Ruiz, Pardo y San Martín, Batista y Coenders. ${ }^{5,23-25}$ Otros como Casas ${ }^{2}$, utilizan SEM para determinar el índice de satisfacción al cliente en toda Europa, estimando los parámetros del modelo con ecuaciones lineales simultáneas (PLS).

En el área de calidad, Cuautle ${ }^{26}$ utiliza estos modelos para proponer un modelo de gestión integral para las empresas mexicanas de manufactura. Relacionado a la transferencia del conocimiento, tanto Maynez ${ }^{27}$ como Mejía y Cornejo ${ }^{1}$ utilizan estos modelos en sus respectivas investigaciones de doctorado.

En lo que respecta al área educativa, se encuentran investigaciones como la de Cervelló et $\mathrm{al} 2^{8}$, en la que analizan la motivación de los alumnos en las clases de educación física. Briseño, Herrera, Enders y Fernández ${ }^{29}$ los utilizaron para evaluar los riesgos ergonómicos en el personal de enfermería. Lozano ${ }^{30}$ valida un modelo para determinar las dificultades a las que se enfrentan los estudiantes en el momento de decidir qué carrera estudiar. Blázquez, Álvarez, Bronfman y Espinosa ${ }^{31}$ presentan un estudio de los factores que influyen en la motivación de los escolares en las áreas de ingeniería y tecnología entre muchos otros investigadores de diferentes áreas de aplicación.

\section{CONSTRUCCIÓN DE LOS MODELOS}

El análisis factorial se utiliza para reducir y resumir los datos que se están analizando32, eliminando las dependencias o independencias de los datos. De igual manera se utiliza para "identificar factores que explican las correlaciones entre las variables”26, siendo que en este tipo de análisis todas las variables son iguales, o sea independientes, lo que significa que ninguna variable es más o menos que otra. ${ }^{33}$

$\mathrm{Al}$ tener la información requerida, es necesario calcular un conjunto de dimensiones latentes llamadas factores, con los que se explican dichas relaciones. Por lo tanto, el análisis factorial utiliza una técnica de reducción de datos con la que se pueden probar las hipótesis planteadas. ${ }^{7}$

El Análisis Factorial puede ser de dos tipos. El exploratorio, en el que no se conoce de inicio el número de factores y es en la aplicación empírica donde se determina este número. En el análisis confirmatorio, los factores se conocen de entrada, utilizando las hipótesis para su corroboración. ${ }^{4}$

En esta investigación se utilizan los dos tipos de análisis, ya que es preciso conocer la relación que existe entre las variables contenidas en la SL, en los FSC y en los FEA para determinar su impacto en la primera.

Para llevar a cabo el análisis factorial es necesario seguir una serie de pasos, como lo expone Malhotra ${ }^{32}$, en el que primeramente se tiene que hacer la formulación del problema para poder analizar la matriz de correlación. Se debe determinar el número de factores para después rotarlos y extraerlos para su interpretación.

\section{Análisis factorial exploratorio (AFE)}

El AFE permite generar estructuras de modelos teóricos e hipótesis que se puedan contrastar empíricamente ${ }^{33}$, sin tener especificaciones previas del modelo ni considerar tanto el número de factores como la relación entre estos. ${ }^{34}$

La técnica que utiliza el AFE es extraer los factores con cierto criterio estadístico, obteniendo la estructura factorial más simple en cuanto a su interpretación más fácil y significativa. ${ }^{35,36}$

Una vez que se tienen los valores medios de cada constructo, así como su desviación estándar, es necesario analizar la matriz de componentes (Tabla 1), para determinar los items que pertenecen a cada constructo, con la finalidad de establecer el instrumento correcto. Se toma el criterio de aceptar aquellos ítems cuyo valor sea mayor o igual a .5, quedando compuestos como se observa a continuación en la Tabla 1.

Como se muestra en la tabla a continuación, el resultado de la matriz de componentes encuentra que, de los 16 ítems originales, tres no se encuentran dentro de los niveles indicados, descartando

\section{Tabla 1.}

Matriz de componentes por constructo.

\begin{tabular}{|c|c|c|c|}
\hline \multirow[b]{2}{*}{ ITEM } & \multicolumn{3}{|c|}{ Componentes } \\
\hline & SL & FSC & FEA \\
\hline 14 & 0,842 & & \\
\hline 15 & 0,814 & & \\
\hline 11 & 0,744 & $-0,521$ & \\
\hline 18 & 0,739 & & \\
\hline 16 & 0,696 & & \\
\hline 13 & 0,660 & & \\
\hline 17 & 0,658 & & \\
\hline 19 & 0,548 & & \\
\hline \multicolumn{4}{|l|}{12} \\
\hline |11 & & 0,747 & \\
\hline I10 & & 0,723 & \\
\hline \multicolumn{4}{|l|}{ I12 } \\
\hline I15 & & & 0,886 \\
\hline I16 & & & 0,721 \\
\hline I14 & & & 0,719 \\
\hline 113 & & & \\
\hline
\end{tabular}

Fuente: elaboración propia. 
el 2, 12 y 13. Dentro del constructo de satisfacción laboral se elimina el dos agregando el nueve que pertenece al constructo de FSC, quedando al final ocho.

En relación al constructo de FSC, se eliminó el 12, quedando en total dos de los cuatro originales. Respecto a FEA, se quedan tres de los cuatro originales, eliminando el 13 que corresponde a la iluminación natural de la oficina, dejando en claro desde un principio que esta variable no pertenece a los FEA, quedando al final 13 variables de las 16 originales.

\section{Análisis factorial confirmatorio (AFC)}

El AFC permite corregir o corroborar, en caso de haberlas, las deficiencias del AFE, conduciendo a una mayor contrastación de las hipótesis especificadas ${ }^{3}$; de igual manera analiza la matriz de covarianzas en lugar de la de correlaciones, lo que ayuda a establecer si los indicadores son equivalentes. ${ }^{12}$

El AFC se representa mediante diagramas de flujo (path diagram), de acuerdo a sus especificaciones particulares. Los rectángulos representan los items y las elipses, los factores comunes. Las flechas unidireccionales entre los factores comunes y los items expresan saturaciones y las flechas bidireccionales indican la correlación entre factores comunes o únicos. ${ }^{37}$

En conclusión, estos modelos facilitan el marco estadístico adecuado para evaluar la validez y confiabilidad de cada ítem en lugar de realizar un análisis global ${ }^{25}$, ayudando al investigador a optimizar tanto la construcción de un instrumento de medición como el análisis de resultados.

En el AFC es necesario observar las cargas factoriales que permiten establecer la correlación entre las variables y los factores. ${ }^{38}$ Entre más se acerquen a uno, mayor será dicha correlación. Una regla empírica en el AFC establece que las cargas deben ser $\geq$ a 0,07 . Pese a que es alto y algunos factores y variables pueden quedar fuera del modelo, estos deben tomarse a criterio del investigador. ${ }^{39}$

Tomando los criterios de Garson, Raubenheimer y Widaman ${ }^{37-39}$ en los que establecen que, para eliminar al máximo la colinealidad entre variables, es necesario tomar las variables cuyas cargas sean $\geq 0,07$, sobre todo tratándose de una propuesta de modelo, en cuyo caso su construcción se basa en la teoría existente.

Para el caso particular de esta investigación, el AFC queda como se muestra en la Figura 2, en donde quedan al final nueve variables. I1, I4, I5, I8 pertenecen a la satisfacción laboral, I10 e I11 son del grupo de FSC y las variables pertenecientes a los FEA son I14, I15 y I16.

\section{Figura 2.}

Análisis factorial confirmatorio.

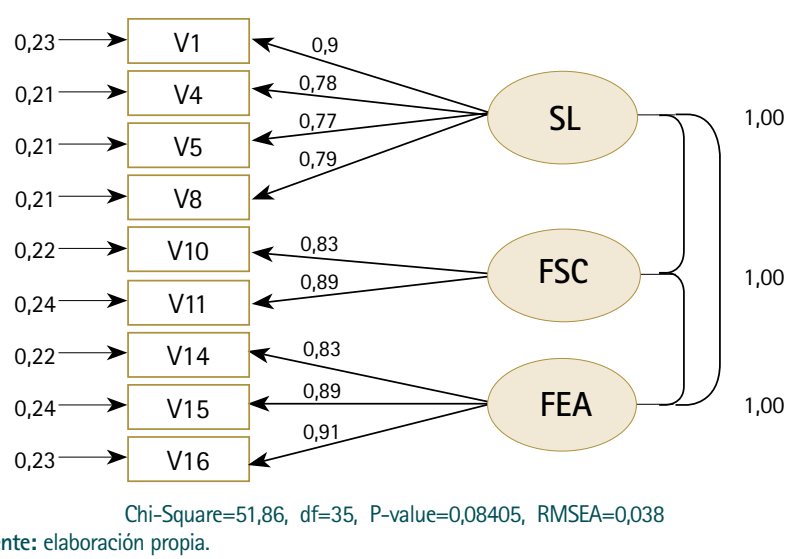

De un total de 16 variables que se tienen en el AFE, se eliminan cuatro más por tener cargas < a 0,0738, siendo estas la 2, 3, 6, 7, 9, 12 y 13, quedando al final nueve variables con las que se analizan las hipótesis para determinar si se aceptan o se rechazan.

Teniendo los valores del AFC, se deben calcular los valores entre las hipótesis para determinar si se aceptan o se rechazan, las mismas que se observan en la Figura 1 como $\gamma 11, \gamma 12, \gamma 21$ y $\gamma 22$ y con las que se pretende establecer la relación entre los constructos del modelo.

\section{Valores estadísticos}

Dado que el modelo presentado tiene variables latentes o no observadas, es necesario identificar cada una de estas con un valor estadístico para poder calcular los estimados de sus efectos. Los valores estimados evalúan un parámetro que caracteriza a la población a través de una muestra. Si esta representa fielmente a la población, se supone que el estadístico representa bien el parámetro. Para que los valores estimados en un modelo sean aceptables, estos deben tener una carga $\geq 0,07.41-43$

Se expone una breve explicación de cada uno de los valores utilizados en la evaluación de los criterios de calidad de ajuste, evaluando desde tres perspectivas: ajuste absoluto, ajuste incremental y ajuste de parsimonia.

\section{Tipos de valores estadísticos}

Bondad de ajuste absoluto: Determina el grado en que el modelo general predice la matriz de correlaciones y para SEM, el estadísticoradio de verosimilitud Chi-cuadrado es la única medida estadística. ${ }^{14}$ El modelo tiene un ajuste aceptable si los valores de Chi-cuadrado/gl son de 2 a 3 y con límites de hasta $5 .^{18}$

Cuando se tiene una muestra pequeña, se utiliza el parámetro de No centralidad (NCP), la cual es una medida alternativa de Chi-cuadrado, en donde los valores menores a 2 son aceptables. ${ }^{44}$

El Índice de bondad de ajuste (GFI) evalúa si el modelo debe ser ajustado. Entre más se acerque a cero indica un mal ajuste. ${ }^{45}$

El Error de Aproximación Cuadrático Medio (RMSEA), representa el ajuste anticipado con el valor total de la población y ya no con el de la muestra. ${ }^{46} \mathrm{Si}$ RMSEA es menor o igual a 0,05 indica un error de aproximación del modelo con la realidad. ${ }^{14}$

El índice de error de cuadrático medio (RMR) mide las varianzas y covarianzas de la muestra y si éstas difieren de las estimaciones obtenidas. ${ }^{16} \mathrm{Si}$ este indicador se acerca a 0 , puede considerarse un ajuste casi perfecto. ${ }^{48}$

El índice de validación cruzada esperada (ECVI) representa la correlación entre las variables del modelo. Entre más se acerque a $1 \mathrm{su}$ correlación es mayor. ${ }^{14}$

Medidas de ajuste incremental del modelo: estas medidas comparan el modelo propuesto con algún otro existente, llamado generalmente modelo nulo. Los índices para evaluarlo se exponen a continuación. El Índice ajustado de bondad de ajuste (AGFI) es una extensión del GFI, el cual ajusta los grados de libertad entre los dos modelos. Los valores cercanos a 0,90 o superiores son los que muestran un mejor ajuste del modelo. ${ }^{44}$

El índice No normalizado de ajuste (NNFI), o indice Tucker Lewis, supera las limitaciones del NFI al considerar los grados de libertad del modelo propuesto, siempre y cuando su relación sea débil con el tamaño muestral. El rango de este va de entre 0 y 1 , siendo recomendables los valores superiores o iguales a 0,9.47

El índice normado de ajuste (NFI), compara el modelo propuesto y el modelo nulo considerando un valor aceptable si es mayor a $0,90 .{ }^{46,48}$ Medidas de ajuste de parsimonia: estas relacionan la calidad de 
ajuste del modelo con el número de coeficientes estimados necesarios para conseguir el nivel de ajuste. Los valores se congregan en tres grupos, bajos, adecuados o elevados dependiendo de su valor, ya que este oscila entre 0 y 1.

El Índice de ajuste normado de parsimonia (PNFI) relaciona los constructos con la teoría que los sustenta. Entre más cerca esté de 1.0 es mayor su relación. ${ }^{45}$

El Criterio de información de Akaike $^{49}$ (AIC) es una medida comparativa entre modelos con diferente número de constructos. Los valores cercanos a 0 indican un mejor ajuste y una mayor parsimonia, teniendo que el modelo es mejor por ser una medida comparativa.

El Índice de bondad de ajuste de parsimonia (PGFI) es un índice sugerido por Mulaik y colaboradores ${ }^{50}$, que constituye una modificación del GFI y considera los grados de libertad disponibles para probar el modelo. Las magnitudes consideradas aceptables se encuentran en el rango de 0,5 a 0,7.

\section{RESULTADOS DE LOS VALORES ESTADÍSTICOS}

Como criterio para obtener el modelo final (Figura 3), se dejan aquellas variables cuya carga en valores estimados sea $\geq 0,07.41-43$ Tomando los resultados obtenidos por el LISREL, se obtienen las medidas de bondad de ajuste como se observa en la Tabla 2.

En el modelo final, las variables contenidas en la SL corresponden a cómo se siente el trabajador con respecto a la libertad de acción, a la relación con su jefe, a las promociones que puede obtener, a la gestión de la empresa y a cómo se le toma en cuenta respecto a las sugerencias que hace.

Las variables respectivas de los FSC están relacionadas con el lugar de nacimiento, el estado civil, el lugar de procedencia y los dependientes económicos. Y en cuanto a los FEA, la iluminación artificial y la temperatura dentro de las oficinas son variables directamente relacionadas.

\section{Pruebas de hipótesis}

Al trabajar con modelos de ecuaciones estructurales, se tiene la ventaja de comprobar todas las hipótesis al mismo tiempo, ya que se analiza el comportamiento de cada una de las variables contra las demás. ${ }^{6}$

Como se observa en la Figura 3, se tienen los valores de cada grupo de factores, las cargas de las variables y el valor obtenido, por

Figura 3.

Valores del modelo general.

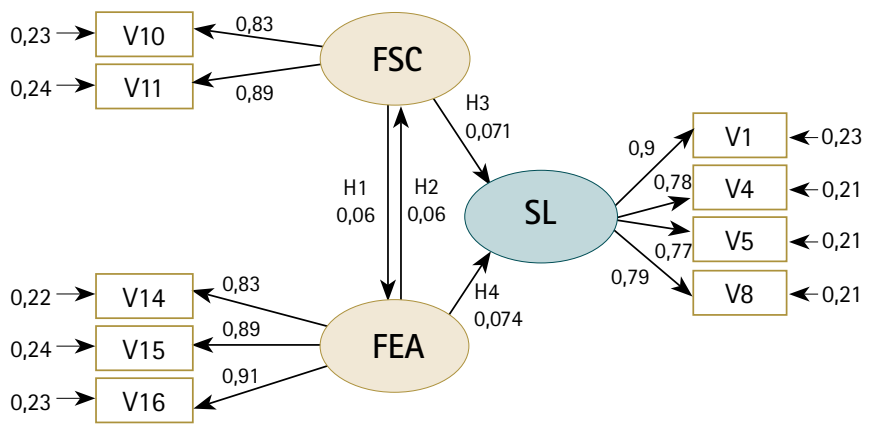

Chi-Square $=51,86, d f=35$, P-value $=0,8405$, RMSEA $=0,038$ Fuente: elaboración propia.
Tabla 2.

Medidas de bondad de ajuste.

\begin{tabular}{|c|c|c|c|}
\hline $\begin{array}{l}\text { Medida de bondad } \\
\text { de ajuste }\end{array}$ & $\begin{array}{l}\text { Niveles de ajuste } \\
\text { aceptable }\end{array}$ & $\begin{array}{l}\text { Aceptabilidad } \\
\text { obtenidos }\end{array}$ & Valores \\
\hline \multicolumn{4}{|l|}{ Medidas de ajuste absoluto } \\
\hline $\begin{array}{l}\text { Estadistico ratio de } \\
\text { Verosimilitud }\end{array}$ & & $X^{2}=51,86$ & Bajo \\
\hline Chi-cuadrado & & $P$ value $=0,084$ & Aceptable \\
\hline $\begin{array}{l}\text { Parámetro de No } \\
\text { centralidad (NCP) }\end{array}$ & $\begin{array}{l}\text { Tomados de } \\
\mathrm{X}^{2}\end{array}$ & 12,86 & No aceptable \\
\hline $\begin{array}{l}\text { Indice de bondad de } \\
\text { Ajuste (GFI) }\end{array}$ & $\begin{array}{c}0 \text { mal ajuste; } \\
1.0 \text { ajuste perfecto }\end{array}$ & 0,91 & Aceptable \\
\hline $\begin{array}{l}\text { Error de aproximación } \\
\text { cuadrático medio (RMSEA) }\end{array}$ & $<=0,05$ & 0,038 & Aceptable \\
\hline $\begin{array}{l}\text { Indice de error cuadrático } \\
\text { Medio (RMR) }\end{array}$ & Cerca de 0 & 0,03 & Aceptable \\
\hline $\begin{array}{l}\text { İndice de validación } \\
\text { cruzada esperada (ECVI) }\end{array}$ & $\begin{array}{l}\text { Entre más cerca de } 1 \text { su } \\
\text { correlación aumenta }\end{array}$ & 0,82 & Aceptable \\
\hline $\begin{array}{l}\text { Medidas de ajuste incremen } \\
\text { Índice normado } \\
\text { de ajuste (NFI) }\end{array}$ & $>0,90$ & 0,92 & Aceptable \\
\hline $\begin{array}{l}\text { Índice No normalizado } \\
\text { de ajuste ó Tucker Lewis } \\
\text { (NNFI/TLI) }\end{array}$ & $>=0,90$ & 0,95 & Aceptable \\
\hline $\begin{array}{l}\text { Índice ajustado de bondad } \\
\text { de ajuste (AGFI) }\end{array}$ & $>=0,90$ & 0,90 & Aceptable \\
\hline $\begin{array}{l}\text { İndice de bondad de } \\
\text { ajuste (GFI) }\end{array}$ & $>=0,90$ & 0,91 & Aceptable \\
\hline $\begin{array}{l}\text { Medidas de ajuste de parsim } \\
\text { Índice de bondad de ajuste } \\
\text { De parsimonia (PGFI) }\end{array}$ & $\begin{aligned} & A>\text { valor } \\
> & \text { parsimonia }\end{aligned}$ & 0,55 & Aceptable \\
\hline $\begin{array}{l}\text { İndice de ajuste normado } \\
\text { de parsimonia (PNFI) }\end{array}$ & A > valor < ajuste & 0,67 & Ajuste medio \\
\hline Chi-cuadrado normada & $\mathrm{LI}=1,0 ; \mathrm{LS}=2,3$ ó 5 & 0,06 & Bajo \\
\hline $\begin{array}{l}\text { Criterio de información } \\
\text { de Akaike (AIC) }\end{array}$ & $\begin{array}{l}\text { Valor pequeño } \\
\text { Indica parsimonia }\end{array}$ & 1104,72 & Bajo \\
\hline
\end{tabular}

Fuente: elaboración propia.

medio del paquete computacional LISREL, de las cuatro hipótesis pertenecientes a esta investigación.

Se tiene que P-value es la probabilidad de obtener un resultado que suponga que la hipótesis nula $\left(\mathrm{H}_{0}\right)$ sea cierta. ${ }^{51}$ Para rechazar esta hipótesis se tiene que asociar el resultado observado con el valor $\mathrm{p}$, por lo tanto, el valor $\mathrm{p}$ oscila entre $0 \mathrm{y} 1$ por lo que los valores altos NO rechazan la hipótesis nula.

Dado que el valor $P$ en el modelo general es igual a 0,08405 y el Error de Aproximación Cuadrático Medio (RMSEA) $=0,038$, y siendo que el primero debe ser $\geq 0,05$ y el segundo $\leq 0,05$, se establece que las relaciones entre los constructos y las hipótesis tienen significancia. De lo anterior se analizan por separado las hipótesis.

Comenzando con la hipótesis 1, se tiene que los FSC tienen una magnitud del 0,06 sobre el efecto de los FEA. Para determinar si los grupos de factores anteriores tienen impacto sobre la SL, se establece un efecto significativo de los FSC hacia la SL de 0,071, siendo > 0,05 (valor mínimo recomendado), y un efecto significativo del 0,074 (< 0,05 valor mínimo recomendado) de los FEA a la SL. Aunado a lo anterior se tiene un RMSEA $=0,038$, ( $\leq$ 0,05 valor máximo recomendado) y un valor del índice Tucker-Lewis $=0,95$ ( $>$ 0,90 valor mínimo recomendado), por lo que no se rechaza la hipótesis:

H1: Los FSC tienen relación con los FEA que impactan en la SL La variables más importantes que explican los FSC y FEA son V11 
con un valor estimado de 0,89 y V16 con un valor estimado de 0,91 , respectivamente.

Con los mismos valores de la hipótesis anterior se tiene un efecto significativo entre los FEA y los FSC, dado que su valor estimado es de 0,06 y su impacto con la SL es del 0,074 y 0,071 respectivamente, por lo que no se rechaza la hipótesis:

H2: Los FEA tienen relación con los FSC que impactan en la SL

Para establecer la relación, ya sea positiva o negativa entre los FSC y la SL, se analiza la hipótesis 3 cuyo valor estimado $=0,071$ (> 0,05 valor recomendado), con un valor $\mathrm{P}=0,08405$ y un índice de Tucker-Lewis $=0,95$ se determina una relación positiva, no se rechaza la hipótesis:

H3: Existe una relación directa de los FSC con la SL

Dado que se tiene un efecto significativo entre los FEA y la $\mathrm{SL}=$ 0,074 ( $\geq 0,05$ valor recomendado), un valor $\mathrm{P}=0,08405$ y indice Tucker-Lewis $=0,95$, no se rechaza la hipótesis:

H4: Existe una relación directa de los FEA con la SL.

\section{CONCLUSIONES}

Durante el desarrollo de este trabajo se pudieron efectuar varios propósitos, tales como exponer tanto las definiciones, características, fases, aplicación y construcción de los modelos de ecuaciones estructurales. De igual manera, mostrar los paquetes computacionales estadísticos con los que se puede diseñar, analizar y construir un modelo. Aunado a lo interior, este trabajo brindó los aspectos necesarios para diseñar el modelo propuesto de un caso real y que detalla cada uno de los pasos del mismo, mostrando el modelo final resultante de la investigación aplicada al personal académico del Departamento de Ingeniería Industrial y Manufactura de una Institución de Educación Superior en Ciudad Juárez, Chihuahua.

Asimismo se observa que para este tipo de modelos -los cuales contienen muchas variables y en su análisis se tiene que prestar atención a la relación que hay entre una de ellas contra las otras-, los modelos de ecuaciones estructurales constituyen una de las mejores opciones, señalando que para el presente ejemplo se utilizó el paquete computacional estadístico LISREL. ${ }^{9}$

Relacionado con los resultados, se observa en el estudio que los valores que determinan si una hipótesis es aceptada o rechazada están dentro de los rangos aceptables, por lo que las cuatro hipótesis son aceptadas; siendo estas:

H1: Los FSC tienen relación con los FEA que impactan en la SL con valor de 0,06 .

H2: Los FEA tienen relación con los FSC que impactan en la SL con valor de 0,06.

H3: Existe una relación directa de los FSC con la SL con valor de 0,071 .

H4: Existe una relación directa de los FEA con la SL con valor de 0,074

Para finalizar y tomando en cuenta el nivel de error con el que se trabajó en esta investigación, los resultados comprueban la relación entre las variables y los constructos en estudio, siendo los FEA los que más impactan en la SL, y en mínima relación con esta variable son los FSC. Este hallazgo puede tener grandes beneficios tanto para el trabajador en su salud y bienestar como para las instituciones en los efectos de productividad de su personal, al crear estrategias que conjunten estos tres grandes factores. 
1. Mejia M, Cornejo C. Aplicación del modelo de ecuaciones estructurales a la gestión del conocimiento. Arequipa: LACCEl; 2010.

2. Casas M. Los modelos de ecuaciones estructurales y su aplicación en el índice europeo de satisfacción al cliente. Madrid: Universidad San Pablo-CEU; s.a.

3. Bollen KA. Structural equations with latent variables. New York: John Wiley \& Sons; 1989.

4. Jöreskog K. Modelado de ecuaciones estructurales. Euskadiko: Guipúzcoa; 1974.

5. Mateos $G$, Morales A. Los modelos de ecuaciones estructurales: una revisión histórica sobre sus origenes y desarrollo. En: Historia de la Probabilidad y la Estadistica V. Riobóo Almanzor JM, Riobóo Lestón I, editores. Santiago de Compostela: AHEPE; 2011. p. 289-301

6. Chin W. Issues and opinion on Structural Equation Modeling. MIS Quarterly.1998; 22(1):00

7. Afifi A, Clark V. Computer-aided multivariate analysis. New York: Chapman \& Hall; 1990.

8. Cea M. Analysis multivariable. Teoria y práctica en la investigación social. Madrid: Síntesis; 2002.

9. Jöreskog K, Sörbom D. LISREL 8: User's reference guide. Chicago: Scientific Software International; 1996.

10. Fornell C. A second generation of multivariante analisys, an overview. In: Fornell C. A second generation of multivariante analisys. New York: Plaeger Publishers; 1982. p. 1-21.

11. Haenlein $M$, Kaplan $A$. A begginner's guide to partial least squares analysis. Und statistics. 2004;3(4):283-297.

12. Batista J, Coenders G. Modelos de ecuaciones estructurales. Madrid: La Muralla; 2000.

13. Kaplan D. Structural equation modeling: Foundations and extensions. Newbury Park: Sage; 2000.

14. Kline R. Principles and practice of structural equation modeling. New York: Gilford Press; 2005.

15. Cupani M. Análisis de Ecuaciones Estructurales: conceptos, etapas de desarrollo y un ejemplo de aplicación. Tesis. 2012; 2(1):186-199.

16. Arbuckle J. Amos user's guide. Chicago: SmallWaters; 2003.

17. Bentler, P. 1995. EOS structural equations program manual. Encino, CA: Multivariate Software.

18. Hair J, Anderson R, Tatham R, Black W. Análisis Multivariante. $5^{a}$ ed. Madrid: Prentice Hall; 1999.

19. Hu L, Bentler P. Fit indices in covariance structure modeling: Sensitivity to underparameterized model misspecification. Psychol Methods. 1998;3:424453.

20. Tabachnick B, Fidell L. Using multivariate statistics. New York: Harper \& Row; 2001.

21. Pardo A, Ruiz M. SPSS 11. Guia para el análisis de datos. Madrid: McGraw-Hill; 2002.

22. Camacho J. Estadistica con SPSS para Windows: Version 12. México: Alfaomega; 2006.

23. Silva C, Schiattino I. Modelos de ecuaciones estructurales ¿Qué es eso? Cienc trab. 2008; 10(29):106-110.

24. Ruiz $M$, Pardo A, San Martín R. Modelos de ecuaciones estructurales. 2010;31(1):34-45.

25. Batista J, Coenders G, Alonso R. Introducción a los modelos estructurales. Utilización del análisis factorial confirmatorio para la depuración de un cuestionario. En: Renom J, editor. Tratamiento informatizado de datos. Barcelona: Masson; 2004. p. 229-286.

26. Cuautle L Creación de una propuesta de modelo de sistema de gestión integral para las empresas mexicanas de manufactura [Tesis doctoral]. Puebla: Universidad Popular Autónoma del Estado de Puebla; 2010.

27. Maynez A. La transferencia de conocimiento organizacional como fuente de ventaja competitiva sostenible: modelo integrador de factores y estrategias. [Tesis doctoral]. Puebla: Universidad Popular Autónoma del Estado de Puebla; 2011.

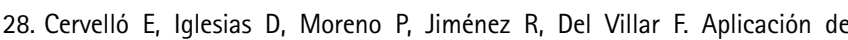
modelos de ecuaciones estructurales al estudio de la motivación de los alumnos en las clases de educación física. Rev Educ (Madrid). 2004; (335):371-383.

29. Briseño $C$, Herrera R, Enders J, Fernández A. Estudio de riesgos ergonómicos y satisfacción laboral en el personal de enfermería. Rev Salud Pública (Córdoba). 2005;9(1):53-59.

30. Lozano S. Validación de un modelo de medida de las dificultades en los procesos de toma de decisiones sobre la carrera profesional. Rev Educ (Madrid). 2007;(343):325-351.

31. Blázquez C, Álvarez P, Bronfman N, Espinosa J. Factores que influencian la motivación de escolares por las áreas tecnológicas e ingenieria. Calidad en la educación. 2009;(31):46-64.

32. Malhotra N. Investigación de mercados. México: Prentice Hall; 1997.

33. Gorsuch R. Factor Analysis. 2nd ed. Hillsdale, NJ: Erlbaum; 1983.

34. Gerbin D, Hamilton J. Viability of Exploratory Factor Analysis as a Precursor to Confirmatory Factor Analysis. Struct Equ Modeling. 1996;3(1):62-72

35. Thurstone L. Multiple Factor Analysis. Psychol Rev. 1931;38:406-427.

36. Thurstone L. Multiple Factor Analysis. Chicago, IL: University of Chicago Press; 1947.

37. Jöreskog K. A general approach to confirmatory maximum likelihood factor analysis. Psychometrika. 1969;34:183-202.

38. Garson D. Factor Analysis. Asheboro: North Caroline State University Press; 2013. (Blue Books Serie).

39. Raubenheimer JE. An item selection procedure to maximize scale reliability and validity. S Afr J Ind Psychol. 2004;30(4):59-64.

40. Widaman KF. Common factor analysis versus principal component analysis: Differential bias in representing model parameters? Multivar Behav Res. 1993;28:263-311.

41. Blume J. Aplicación de estadística para ingenieros. Barcelona: Labor; 1976.

42. Hulland J. Use of partial least squares (PLS) in strategic management research: a review of four recent studies. Strateg Manage J. 1999; 20(2):195-204.

43. Barclay D, Higgins C, Thompson R. The Partial Least Squares (PLS) Approach to Causal Modelling: Personal Computer Adoption and Use as an Illustration. Technol Stud (Spec Iss Res Methodol). 1995;2(2):285-309.

44. Martínez M. Orientación al mercado. Un modelo desde la perspectiva de aprendizaje organizacional. México: Planeta; 2003.

45. Torres $\mathrm{G}$. Una aproximación al análisis de competitividad aplicando la técnica del modelo de ecuaciones estructurales. TESCoatl [en línea]. 2011 [citado nov 2015]; 13(31):1-9.Disponible en: http://www.tesco.edu.mx/gem/DOC/PDF/ publi- caciones/tescoatl/tesco_pdf_tescoat|31_4_analisiscompetitividad.pdf

46. Lévy JP. Modelización y análisis con ecuaciones estructurales. En: Lévy JP, Varela J, editores. Análisis Multivariante para las Ciencias Sociales. Madrid: Prentice Hall. 2003. p.769-810.

47. Lévy JP, Varela J. Modelación con estructuras de covarianzas en ciencias sociales. Madrid: Netbiblo; 2006.

48. Byrne BM. Structural equation modeling with AMOS. Basic concepts, applications and programming. Mahwah, NJ: Lawrence Eribaum Associates; 2001.

49. Akaike H. Factor analysis and AIC. Psychometrika. 1987;52(3):317-332.

50. Mulaik S, James L, Van Alstine J, Bennet N, Lind S, Stilwell C. Evaluation of goodness of fit indices for structural equation models. Psychol Bull. 1989;105(3):430-445.

51. Nava R, Mercado P. Análisis de trayectoria del capital intelectual en una universidad pública mexicana. Redie. 2011;13(2):166-187.

\section{Referencia complementaria:}

Del Barrio S, Luque T. Análisis de ecuaciones estructurales. En: Luque T. Técnicas de Análisis de Datos en Investigaciones de Mercados. Madrid: Edcs. Pirámide; 2000. p. 489-552. 